\title{
Usefulness of Radial Artery as a Carotid Artery Patch in Simultaneous Carotid Endarterectomy and Coronary Artery Bypass Graft Operation with Complete
} Arterial Revascularization

\author{
Abdussamet Hazar, Assoc. Prof., ${ }^{1}$ Özgür Altınbaş, Asst. Prof. Dr., ${ }^{2}$ Mehmet Salih Aydın, Assoc. Prof., ${ }^{3}$ \\ Aydemir Koçarslan, Assoc. Prof. Dr. ${ }^{4}$ \\ ${ }^{1}$ Department of Cardiovascular Surgery, Dr. Konya Training and Research Hospital, Konya, Turkey; ${ }^{2}$ Department of Operating \\ Room Services, Gaziantep University, Vocational School of Health Services, Gaziantep, Turkey; ${ }^{3}$ Department of Cardiovascular \\ Surgery, Dr. Harran University, Şanlıurfa, Turkey; ${ }^{4}$ Department of Cardiovascular Surgery, Kahramanmaraş Sütçü Imam University, \\ Kahramanmaraş, Turkey
}

\section{ABSTRACT}

Background: Serious coronary artery diseases including left main coronary artery disease, proximal left anterior descending artery disease, and three-vessel coronary artery disease with carotid artery stenosis are required simultaneous operations. By using complete arterial revascularization technique for coronary artery bypass graft operation, radial artery can be used safely as a patch material for carotid endarterectomy in combined surgery.

Methods: Between 2016 and 2018, 14 patients who had serious coronary artery disease with the stenosis of unilateral carotid artery equal/over $70 \%$ were included in the study. Complete arterial revascularization was performed in all patients and radial artery was used as a patch material in carotid endarterectomy.

Results: All patients were discharged without any complication and carotid artery colored Doppler ultrasound was performed to the patients in the 3 rd months, 6th months, and first year of the operation. There was no restenosis detected.

Conclusion: In conclusion, radial artery is useable for carotid patch angioplasty in patients who underwent simultaneous carotid endarterectomy and coronary artery bypass graft operation with complete arterial revascularization. Further studies including a large number of patients are needed to examine the long-term patency of this graft.

\section{INTRODUCTION}

Carotid artery endarterectomy (CEA) is the standard management strategy for significant carotid artery stenosis for both symptomatic and asymptomatic patients. Longitudinal arteriotomy is a commonly used surgical approach for CEA but its closure after operation may allow for the possibility

Received fuly 29, 2020; accepted August 27, 2020.

Correspondence: Özgür Altınbaş, Asst. Prof. Dr., Gaziantep University, Medical Faculty, Department of Cardiovascular Surgery, Gaziantep, Turkey; +90505-657-05-77; (e-mail: ozgur_altinbas@yahoo.com). of narrowing the artery. Closure of the arteriotomy by using patches is useful for preventing the neointimal hyperplasia and scarring and allows the steadiness of arterial lumen diameter after procedure.

Patch angioplasty after carotid endarterectomy with different materials like autogenous vein, Dacron and Polytetrafluoroethylene (PTFE) grafts is believed to decrease the incidence of recurrent stenosis compared to primary closure of arteriotomy [Muto 2009]. Beside these, any other materials like bovine pericardium are available in literature as patch material after CEA, while radial artery is used in intracerebral vascular bypass [Hines 2007; Ono 2018].

Although novel improvements and advancement in techniques have been made for carotid stenting, patients with life-threatening coronary artery diseases such as left main coronary artery (LMCA) stenosis, proximal left anterior descending (LAD) coronary artery stenosis or severe threevessel (LAD artery, circumflex artery and right coronary artery) disease may not tolerate hypotension or bradycardia during this procedure. So patients with coronary artery disease concomitant carotid artery disease not suitable for stenting may need combined surgical procedures [Levy 2012].

This study is unique in the literature in terms of radial artery usage after CEA as a patch material, and we aimed to show that radial artery can be used safely as a carotid patch in patients undergoing simultaneous carotid endarterectomy and coronary artery bypass graft operation (CABG) with complete arterial revascularization.

\section{MATERIALS AND METHODS}

Between 2016 and 2018, 14 patients (male, $\mathrm{n}=8$; female, $\mathrm{n}=$ 6 ) with both serious coronary artery disease (LMCA stenosis, $n$ $=3$; proximal LAD coronary artery stenosis, $n=4$; severe threevessel disease, $n=7$ ) and unilateral carotid artery stenosis equal or over $70 \%$ were included in the study. Patients were symptomatic in terms of carotid artery stenosis with the symptoms of dizziness and imbalance. Informed consent was given before operation by all patients. All the patients were equal or over 65 years (min 65 years; max 76 years; mean $67.5 \pm 3.0$ years). Eight 


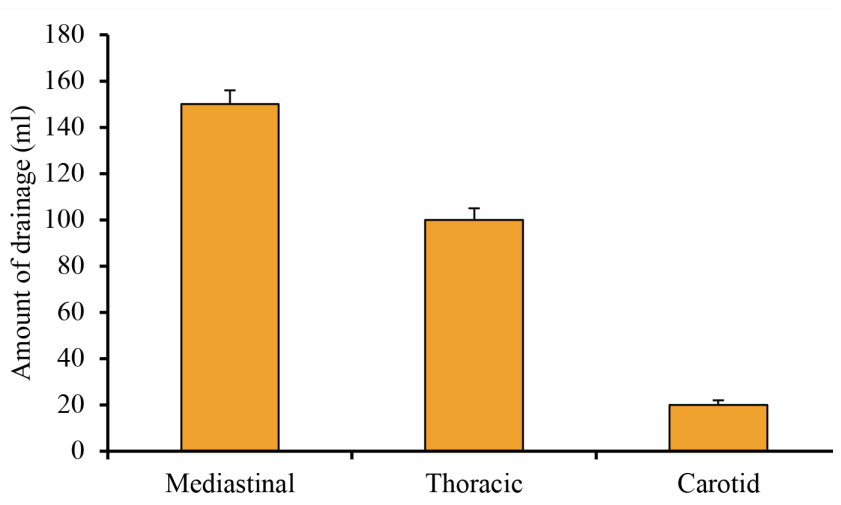

Figure 1. Intraoperative sight of radial artery patch on left internal carotid artery.

of the patients had hypertension (HT), three of them had diabetes mellitus (DM), and three of them had both HT and DM. All patients were taking regular medication due to these diseases. There were no extra chronic conditions in the patients beside these. Exclusion criteria were: redo surgery (both CABG and CEA), bilaterally serious carotid artery stenosis, previous carotid artery stenting procedure, cardiac valve surgery with CABG, chronic diseases like cancer and bleeding disorders, positive result in Allen test, use of saphenous vein as a bypass graft and off-pump surgery. Complete arterial revascularization was performed by using left internal mammary artery (LIMA) and left radial artery (all the patients' use their right hands in their routine lives). Decision of the radial artery usage was given after the physical examination and negative result of Allen test.

CEA was first performed under general anesthesia by maintaining accurate surgical position. Shunt was not used in the operation because retrograde flow of the internal carotid arteries were sufficient for the operations and 6/0 prolene suture was used for replacement of arterial patch. Average carotid cross-clamp time was 20 minutes. This process was followed by on-pump CABG with median sternotomy. A part of the radial artery $(1-2 \mathrm{~cm})$ was used as a patch material at the end of the CEA (Figure 1). Technique of sequential anastomosis was performed in patients with serious three-vessel disease. One drain was placed to carotid loge, one was placed to mediastinal loge, and one was placed to left thoracic loge after operations.

Data regarding duration of operations, intubation, intensive care, service and discharge, medication after operations, complications, and amounts of drainage were recorded.

Carotid artery colored Doppler ultrasound was performed to the patients in the $3 \mathrm{rd}$ month, 6th month, and first year of the operation.

\section{RESULTS}

Characteristic features of all patients are given in the Table. Hypertension was the most accompanied disease $(\mathrm{n}=$ 11) among the patients. Left-sided carotid artery stenosis was significant $(n=11)$ in patients. LMCA disease was more likely
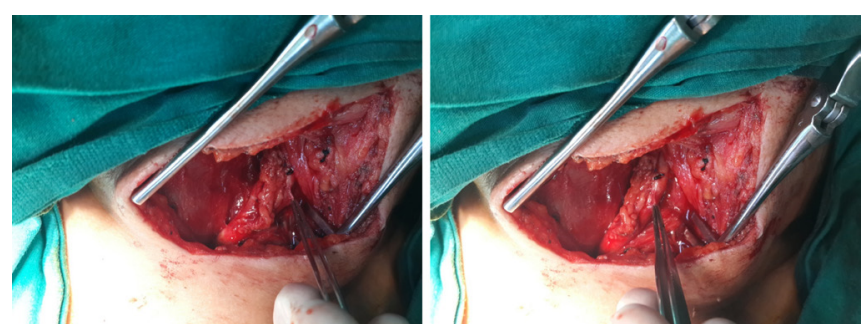

Figure 2. Amount of mean drainage from drains. $P<.001$ for comparison between each drain according to ANOVA with post hoc StudentNewman-Keuls test.

seen in patients with the carotid stenosis over $90 \%$. Mean operation time was $156 \pm 12$ minutes and mean cardiopulmonary pump time was $46 \pm 5$ minutes.

There were no complications include stroke, major bleeding, hematoma, wound and systemic infection detected. There was no excitus.

All patients required positive inotropic support including noradrenaline (range $0.05-0.1 \mu \mathrm{g} / \mathrm{kg}$ ) on day 0 of operation. Support was stopped gradually on postoperative day 1. Four of the patients were extubated on the operation day and others on postoperative day 1. Enoxaparin sodium was administered to the patients $(1 \mathrm{mg} / \mathrm{kg})$ at the 8 th hour of the operation, subcutaneously.

Acetylsalicylic acid (100 mg/day) and clopidogrel $(75 \mathrm{mg} /$ day) were started as an oral antiplatelet therapy and bisoprolol ( $5 \mathrm{mg} /$ day, orally) was started to control the rhythm and arterial blood pressure on the postoperative first day.

Amount of mean drainage are given in Figure 2. According to BART (The Blood Conservation Using Antifibrinolytics in a Randomized Trial) and BRiSc (Bleeding Risk Score) studies that were related to postoperative indices for massive or excessive bleeding, there was no massive or excessive bleeding detected.

Four of the patients stayed two days and ten of the patients stayed three days in the intensive care unit. All patients were discharged at the end of the eighth days after operation.

Patients were called for postoperative control in their first week, first month, third month, sixth month, and first year after operation. Carotid artery colored Doppler ultrasound was performed on the patients in the 3rd months, 6th months, and first year of the operation. No restenosis was detected.

\section{DISCUSSION}

Incidence of carotid artery stenosis is $6 \%$ to $8 \%$ of all patients undergoing $\mathrm{CABG}$, and is related to an increased risk of intraoperative or postoperative stroke. Concomitant carotid artery disease to coronary artery disease is managed in various ways: carotid artery stenting or CEA, either simultaneously with, before, or after CABG [Weimar 2017]. Trachiotis et al declared that simultaneous CEA and CABG is the preferred surgical approach in high-risk patients in terms of coronary artery disease [Trachiotis 1997]. LMCA, proximal LAD, and three-coronary artery vessel diseases are the 
Patients' Clinical Features

\begin{tabular}{ll}
\hline Parameters & Patients $(\mathrm{n}=14)$ \\
\hline Age, $y$ & $67.5 \pm 3.0$ \\
Sex $(\mathrm{n}, \%)$ & \\
Male & $8(57.1)$ \\
Female & $6(42.9)$ \\
Comorbidity (n, \%) & \\
HT & $8(57.1)$ \\
DM & $3(21.4)$ \\
HT+DM & $3(21.4)$ \\
Degree and site of carotid stenosis (n, \%) & \\
70\%-left & $5(35.7)$ \\
$70 \%$-right & $3(21.4)$ \\
$90 \%-l e f t$ & $1(7.1)$ \\
Over 90\%-left & $3(21.4)$ \\
$70-90 \%-l e f t$ & $2(14.2)$ \\
Coronary artery disease (n, \%) & \\
Three vessel & $7(50.0)$ \\
LMCA & $3(21.4)$ \\
Proximal LAD & $4(28.6)$ \\
\hline
\end{tabular}

Data are given as mean \pm SD or $\mathrm{n}(\%)$.

main disorders that threaten the lives of patients undergoing carotid artery stenting or CEA. Hypotension and/or bradycardia may not be tolerated in those procedures [Levy 2012]. Similarly, Hill et al recommended simultaneous CEA and CABG in patients with coronary artery disease and comorbid severe carotid artery disease in their study designed in Canada [Hill 2005]. Nwakanma et al reported that CEA followed by CABG leads to a higher myocardial infarction rate and CABG followed by CEA is related to higher stroke rates, so performing these two operations simultaneously decreases the risk of myocardial infarction and stroke [Nwakanma 2006]. In a study designed by Gansera et al, it is emphasized that combined CEA and CABG operations have acceptable risk for neurological complications and mortality and beside these, a combined procedure yields a reduction in hospital costs [Gansera 2003]. Despite the risks indicated by Gansera et al, there was no mortality or serious morbidity including neurological complications, massive bleeding, or generalized or localized infection seen in our study population. Continuation of preoperative medical therapy including antiaggregant drugs, absence of patients with obesity (body mass index <30) and chronic obstructive pulmonary disease, acceptable operation duration, and early onset of postoperative antibiotherapy were the probable reasons of this clinical situation.

Complete arterial revascularization is a surgical technique in CABG to improve long-term outcomes in the treatment of coronary artery disease. Harvesting of multiple arterial grafts lead to prolonged operating times and increased trauma. Bilateral internal thoracic arteries, the gastroepiploic artery, and the radial artery are used as conduits in complete arterial revascularization [Wendler 2000]. Radial artery is the longest arterial conduit, is less fragile, and has excellent long-term patency up to $87 \%$ [Barner 2001]. Muscular structure of the radial artery makes it prone to spasm during and after harvesting. Papaverine, a kind of alkaloid obtained from opium or prepared synthetically, is used because of its vasodilator property in this clinical situation.

Carotid endarterectomy is performed to prevent recurrent stroke in patients with carotid artery stenosis. Closure technique of the arteriotomy after CEA is considerable due to the rates of restenosis up to $36 \%$ after primary closure. Thus, alternative techniques to reduce these rates were needed and closure of the arteriotomy with a patch has become a routine part of CEA in recent years [Huizing 2018; Arslan 2016]. Saphenous vein and neck veins (external jugular vein and common facial vein) can be used as autogenous patch materials [Dardik 1997]. Synthetic patches are produced from polyester (Dacron), polytetrafluoroethylene (PTFE), and polyurethane materials. In a study conducted by Bond et al, it was reported that PTFE patches reduced restenosis, stroke, and transient ischemic attack (TIA) after CEA operations, compared to the Dacron patches [Bond 2004]. Beside these data, Grimsley et al published their experience on bovine pericardial patch after CEA, and demonstrated that bovine pericardial patch angioplasty during CEA is associated with a low incidence of both perioperative and midterm adverse outcomes [Grimsley 2001]. In a meta-analysis that compared the different patch materials in CEA (synthetic, venous, and bovine pericardium), results were similar in terms of restenosis, 30-day stroke, TIA, myocardial infarction, neck wound infection, local hematoma, carotid artery thrombosis, cranial nerve injury, long-term stroke incidence, and death [Texakalidis 2018]. In a series of 61 patients, Hines et al declared that there was no residual stenosis or anatomic defects at the end of the CEA procedure with pericardial patch [Hines 2007].

Bleeding, rupture, thrombosis and infection are the most prominent patch-materials related complications [Awad 1989]. Autologous veins cost less than synthetic materials and their manipulation is easier. Their endothelial surface can prevent thrombosis and is more resistant to infection than synthetic material [Arslan 2016]. Because of its arterial structure, endothelial surface of the radial artery is more likely to adapt to carotid artery and this may relate to decreased thrombus formation. It is also more resistant to infection than PTFE and Dacron grafts as a result of its autologous nature, and it is more durable than saphenous vein patches in term of rupture because of its muscular structure.

Radial artery was used as a bypass material between common carotid artery and middle cerebral artery in an infected pseudoaneurysm case after CEA in the literature [Yasuda 2005]. It can also be used in reconstruction of the carotid artery that has giant and/or large aneurysm. Houkin et al reported in their study that 43 patients with intracavernous and paraclinoid giant aneurysm of internal carotid artery 
(ICA) have been treated by reconstruction of ICA by using radial artery, and that there was no late-term occlusion in any of these patients [Houkin 1999]. Complex skull base tumors that incorporate large intracranial vessels are challenging clinical situations and there may be occlusion in main arterial structures that need extracranial (common carotid artery, internal carotid artery, external carotid artery) to intracranial (middle cerebral artery) bypass with total tumor resection. Abdulrauf et al performed this bypass by using radial artery and reported it in their study [Abdulrauf 2005]. Radial artery was also used in the anastomosis between the internal maxillary artery and middle cerebral artery to avoid long cervical incision and maintain a short segment and high flow anastomosis in a patient with giant fusiform internal carotid artery aneurysm [Abdulrauf 2011].

In conclusion, radial artery can be used as a carotid patch material in CEA to minimalize the risk of restenosis due to thrombosis, postoperative infection, and rupture in patients undergoing simultaneous CEA and CABG. Further studies including a large number of patients are needed to examine the long-term patency of this graft.

\section{REFERENCES}

Abdulrauf S. 2005. Extracranial-to-intracranial bypass using radial artery grafting for complex skull base tumors: technical note. Skull Base 15:207-13.

Abdulrauf S, Sweeney JM, Mohan YS, Palejwala SK. 2011. Short segment internal maxillary artery to middle cerebral artery bypass: a novel technique for extracranial-to-intracranial bypass. Neurosurgery 68:804-9.

Arslan C, Besirli K, Arapi B, Gode S, Tuzun H. 2016. Mid-and long-term results of external jugular vein patch palsty in carotid endarterectomy. Turk Gogus Kalp Dama 24:51-5.

Awad IA, Little JR. 1989. Patch angioplasty in carotid endarterectomy. Advantages, concerns, and controversies. Stroke 20:417-22.

Barner HB, Sundt TM, Bailey M, Zang Y. 2001. Midterm results of complete arterial revascularization in more than 1000 patients using an internal thoracic artery/radial artery T graft. Ann Surg 234:447-53.

Bond R, Rerkasem K, Naylor AR, Aburahma AF, Rothwell PM. 2004. Systematic review of randomized controlled trials of patch angioplasty versus primary closure and different types of patch materials during carotid endarterectomy. J Vasc Surg 40:1126-35.

Dardik H, Wolodiger F, Silvestri F, et al. 1997. Clinical experience with everted cervical vein as patch material after carotid endarterectomy. $\mathrm{J}$ Vasc Surg 25:545-53.

Gansera B, Angelis I, Weingartner J, Prauser NP, Spiliopoulos K, Kemkes BM. 2003. Simultaneous carotid endarterectomy and cardiac surgery - additional risk factor or safety procedure? Thorac Cardiovasc Surg 51:22-7.
Grimsley BR, Wells JK, Garrett WV, et al. 2001. Bovine pericardial patch plasty in carotid endarterctomy. Am Surg 67:890-5.

Hill MD, Shrive FM, Kennedy J, Feasby TE, Ghali WA. 2005. Simultaneous carotid endarterectomy and coronary artery bypass surgery in Canada. Neurology 64:1435-7.

Hines GL, Feuerman M, Cappello D, Cruz V. 2007. Results of carotid endarterectomy with pericardial patch angioplasty: rate and predictors of restenosis. Ann Vasc Surg 21:767-71.

Houkin K, Kamiyama H, Kuroda S, Ishikawa T, Takahashi A, Abe H. 1999. Long-term patency of radial artery graft bypass for reconstruction of the internal carotid artery. J Neurosurg 90:786-90.

Huizing E, Vos CG, Hulsebos RG, Akker PJ, Borst GJ, Unlu C. 2018. Patch angioplasty or primary closure following carotid endarterectomy for symptomatic carotid artery stenosis. Surg J (NY) 4:96-101.

Levy E, Yakubovitch D, Rudis E, et al. 2012. The role of combined carotid endarterectomy and coronary artery bypass grafting in the era of carotid stenting in view of long-term results. Interact Cardiovasc Thorac Surg 15:984-8.

Muto A, Nishibe T, Dardik H, Dardik A. 2009. Patches for carotid artery endarterectomy: current materials and prospects. J Vasc Surg 50:206-13.

Nwakanma L, Poonyagariyagon HK, Bello R, Khoynezad A, Smego D, Plestis KA. 2006. Early and late results of combined carotid endarterectomy and coronary artery bypass versus isolated coronary artery bypass. Interact Cardiovasc Thorac Surg 5:159-65.

Ono H, Inoue T, Tanishima T, Tamura A, Saito I, Saito N. 2018. Highflow bypass with radial artery graft followed by internal carotid artery ligation for large or giant aneurysms of cavernous or cervical portion: clinical results and cognitive performance. Neurosurg Rev 41:655-65.

Texakalidis P, Giannopoulos S, Charisis N, et al. 2018. A meta-analysis of randomized trials comparing bovine pericardium and other patch materials for carotid endarterectomy. J Vasc Surg 68:1241-56.

Trachiotis GD, Pfister AJ. 1997. Management strategy for simultaneous carotid endarterectomy and coronary revascularization. Ann Thorac Surg 64:1013-18.

Weimar C, Bilbilis K, Rekowski J, et al. 2017. Safety of simultaneous coronary artery bypass grafting and carotid endarterectomy versus isolated coronary artery bypass grafting. Stroke 48:2769-75.

Wendler O, Hennen B, Demertzis S, et al. 2000. Complete arterial revascularization in multivessel coronary artery disease with 2 conduits (skeletonized grafts and T-grafts). Circulation 102:79-83.

Yasuda H, Kuroda S, Ushikoshi S, et al. 2005. Combined surgical and endovascular treatment of infected pseudoaneurysm after carotid endarterectomy. Neurol Med Chir (Tokyo) 45:37-40. 